\title{
Velocity cross-correlations and atomic momentum transfer in simple liquids with different potential cores
}

\author{
A. Verdaguer and J. A. Padró \\ Departament de Física Fonamental, Universitat de Barcelona, Diagonal 647, 08028 Barcelona, Spain
}

(Received 12 November 1999)

\begin{abstract}
Time correlation functions between the velocity of a tagged particle and velocities of particles within specified ranges of initial separations have been obtained by molecular dynamics simulation. These correlation functions have allowed us to analyze the momentum transfer between particles in different coordination shells. Two simple liquids at very different densities and two purely repulsive potentials with very different softnesses have been considered. The longitudinal correlations, which are the velocity cross-correlations along the initial direction defined by the centers of two given particles, have been calculated separately. It has been proven that these correlations should be attributed to particles both in front of and behind the central one. As with propagating longitudinal modes, they are strongly dependent on the softness of the potential core. Some characteristic features of the velocity correlation functions after the initial rise should be related to nonlongitudinal correlations. It has been shown that velocity cross-correlations between distinct particles cannot only be attributed to the direct interactions among particles, but also to the motions induced by the movement of a tagged particle on their neighbors.
\end{abstract}

PACS number(s): 61.20.Lc, 61.20.Ja, 66.20.+d

\section{INTRODUCTION}

A detailed knowledge of the correlations between the motions of distinct atoms is very useful for a full understanding of the microscopic mechanisms of collective dynamic properties in liquids. Time correlation functions between the initial velocity of a central atom and later velocities of neighboring atoms provide interesting information on the characteristics of the transfer of momentum among particles in a liquid [1]. Though these functions cannot be directly obtained from experiments, they can be calculated from molecular dynamics (MD) simulations. Several papers were devoted to the MD analysis of the time correlations among the velocity of a given particle and later velocities of the immediate neighbors in simple liquids [2-9]. A study of these correlations for particles in different coordination shells was recently performed for both simple liquids at different densities and a molten salt $[10,11]$. One of the aims of the present paper is to extend this study by analyzing in more detail the momentum transfer mechanisms by calculating separately the "longitudinal" velocity cross-correlations, i.e., the velocity cross-correlations along the initial direction defined by the centers of two given particles.

It has been proven that the dynamic properties of liquids, such as the velocity autocorrelation functions and the dynamic structure factors, are strongly dependent on the softness of the potential core $[12,13]$. The velocity crosscorrelations analyzed in this paper are closely related to these properties. On the one hand, there is a clear resemblance between the velocity autocorrelation functions and the corresponding velocity cross-correlation functions with the first shell of neighbors $[1,10]$. On the other hand, the velocity cross-correlations with particles in the different coordination shells are very simple collective properties, which are in the origin of the basic mechanisms for the propagation of collective modes through a liquid. In this study we also investigate the dependence of the velocity cross-correlations on the characteristics of the short-range repulsive forces. To this end, the results obtained for systems differing only in the hardness of their potential cores are compared.

\section{DESCRIPTION OF THE SIMULATED SYSTEMS}

Molecular dynamics simulations have been carried out for systems of 864 particles with the atomic mass of argon $\left(m_{\mathrm{Ar}}=39.95 \mathrm{uma}\right)$ in a cubic box with the usual periodic boundary conditions. Two liquids at the same temperature $(T=120 \mathrm{~K})$ but very different densities have been simulated, i.e., a system $A$ with $\rho=1.4 \times 10^{-2}$ particles $/ \AA^{3}$ and a system $C$ with $\rho=2.4 \times 10^{-2}$ particles $/ \AA^{3}$. These densities are somewhat higher than those corresponding to the critical point and the triple point of Ar, respectively. The temperature is intermediate between these two points.

Two interaction potential models have been considered at each density. The first one is a Lennard-Jones (LJ) potential with the usual parameters for $\operatorname{argon}\left(\sigma_{\mathrm{Ar}}=3.405 \AA, \varepsilon_{\mathrm{Ar}}\right.$ $\left.=119.8 k_{B}\right)$, but it is truncated at its minimum $\left(r_{\text {cut }}\right.$ $=2{ }^{1 / 6} \sigma_{\mathrm{Ar}}$ ). This purely repulsive potential will be termed RLJ. The second one is a very soft repulsive potential $V(r)$ $\propto\left(\sigma_{\mathrm{Ar}} / r\right)^{6}$ that will be named RSS. In this case, the interactions have been truncated at $r_{\mathrm{cut}}=2.4 \sigma_{\mathrm{Ar}}$. The core of RSS is markedly softer than that of RLJ since, due to the contribution of the attractive term in the LJ potential, the repulsive wall of RLJ is steeper than that of a 12th inverse-power soft-core potential with the same $\sigma$. The comparison between the results obtained for systems at the same density but using either RLJ or RSS will allow us to analyze the influence of the softness of the potential core on the considered properties. The two systems at the lower density will be termed RLJA and RSSA and those at the higher density will be termed RLJC and RSSC, respectively.

The structure of liquids at the state $A$ are markedly different from those at the state $C$. However, in the case of systems at the same density but different interaction potentials, the 
structures are quite similar $[10,11]$. Thus the effective atomic radii (and therefore the effective thermodynamic states) for RLJA and RLJC are quite close to those for RSSA and RSSC, respectively, and the discrepancies in their dynamic properties should be mainly associated with the differences in their potential cores.

The resulting velocity autocorrelation functions $[C(t)]$ are markedly dependent on the potential. At a given density, the $C(t)$ functions for RSS show slower initial decays than they do for RLJ. Moreover, the $C(t)$ oscillations for the former potential are much more marked than for the latter. This is in accordance with earlier results for liquid metals and LJ fluids that suggested that the more pronounced oscillations of the $C(t)$ functions for liquid metals should be attributed to the softer potential cores for these liquids [1214]. It should be pointed out that despite the solidlike behavior of the $C(t)$ 's for RSSA and RSSC, these systems are in the liquid phase. Both the shape of the radial distribution functions [12] and the values of the self-diffusion coefficients unambiguously reflect this fact. The self-diffusion coefficients for systems at the states $A$ and $C$ show important differences but in both cases correspond to liquids [13]. The self-diffusion coefficients for the systems at the same state with either the RLJ or the RSS potential are rather similar [13].

\section{VELOCITY CROSS-CORRELATIONS}

The time cross-correlation functions $\left[C_{n}(t)\right]$ between the velocity of a central particle $(i)$ and velocities of particles $(j)$ initially lying within neighboring concentric shells of specified radii $a_{n}$ and $b_{n}$ are defined as $[1,10]$

$$
C_{n}(t)=\left\langle\vec{\nu}_{i}(0) \cdot \vec{\nu}_{j}(t)\right\rangle_{n}\left\langle\nu_{i}^{2}\right\rangle^{-1},
$$

where $\left\langle v_{i}^{2}\right\rangle^{-1}$ is a normalization factor and \langle\rangle$_{n}$ a restricted statistical average;

$$
\begin{aligned}
\left\langle\vec{\nu}_{i}(0) \cdot \vec{\nu}_{j}(t)\right\rangle_{n}= & N_{n}^{-1}\left\langle\vec{\nu}_{i}(0) \sum_{j} \vec{\nu}_{j}(t) \theta\left[r_{i j}(0)-a_{n}\right]\right. \\
& \left.\times \theta\left[b_{n}-r_{i j}(0)\right]\right\rangle
\end{aligned}
$$

where $\theta(x)$ is the step function and \langle\rangle is an ordinary statistical average over the different central particles and different time origins. $r_{i j}(0)$ is the initial distance between the central particle $i$ and the particle $j$ in the $n$ shell. The mean number of particles in the $n$ shell $\left(N_{n}\right)$ is given by

$N_{n}=\sum_{j}\left\langle\theta\left[r_{i j}(0)-a_{n}\right] \theta\left[b_{n}-r_{i j}(0)\right]\right\rangle=4 \pi \rho \int_{a_{n}}^{b_{n}} r^{2} g(r) d r$.

The values of $a_{n}$ and $b_{n}$ for each system were chosen to be equal to the position of the $n$th minima of the corresponding $g(r)$ function. Thus $C_{n}(t)$ measures the velocity crosscorrelations of a central atom with single atoms, which at $t$ $=0$ are within the $n$th coordination shell [10]. We considered the correlations up to the third shell $(n=1,2,3)$. It should be noted that the momentum of the central particle is shared by a larger number of particles when it is transferred to the successive coordination shells. Accordingly, the values
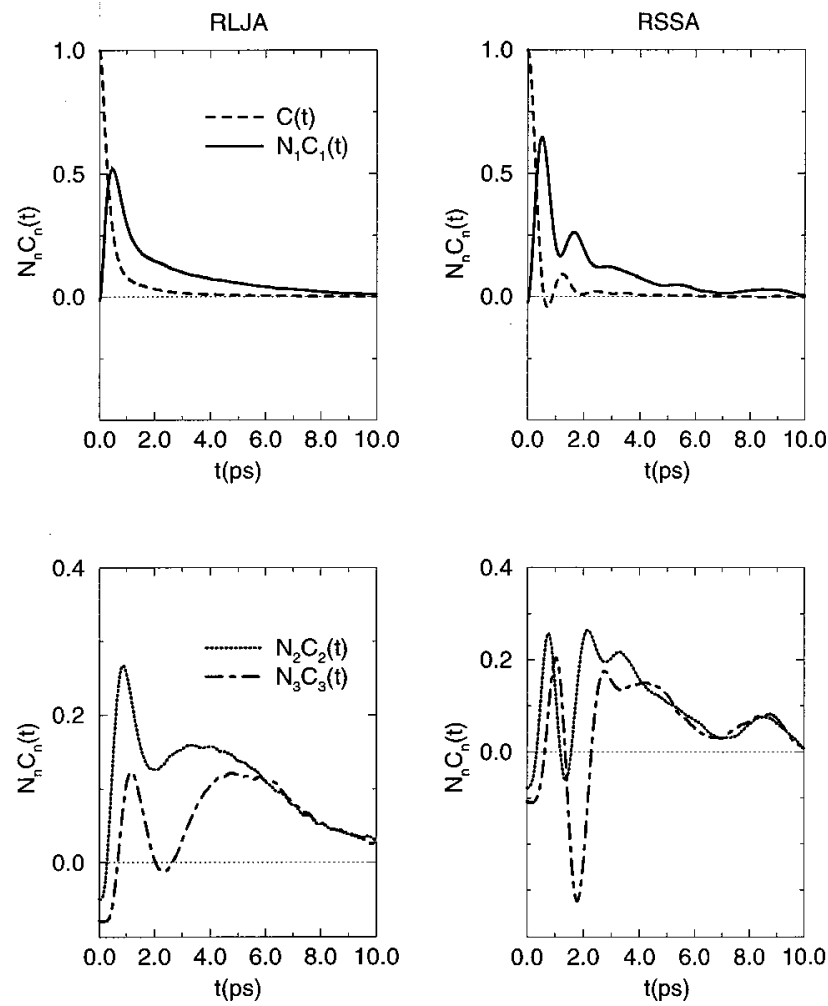

FIG. 1. Velocity cross-correlation functions in the systems at the state $A$ (low atomic density) for atoms within different coordination shells. The velocity autocorrelation functions are also shown in the upper part of the figure.

reached by the $C_{n}(t)$ functions become markedly smaller as $n$ increases. Thus it is useful to consider the $N_{n} C_{n}(t)$ functions, which express the cross-correlations between the velocity of the central particle and the sum of the velocities of all the particles within the $n$th shell of its neighbors. The $N_{n} C_{n}(t)$ functions for the different shells show values of similar order of magnitude [10].

Since the number of particles in the simulation is finite and the total momentum of the cell remains fixed during the MD runs, the $N_{n} C_{n}(0)$ values should be somewhat lower than zero $[3,4,10]$, whereas the $N_{n} C_{n}(t)$ functions corresponding to the actual systems should vanish at $t=0$. These systematic discrepancies in the initial behavior of the $N_{n} C_{n}(t)$ functions should increase with $N_{n}$. However, earlier MD results for systems with different number of particles have shown that, even for $n=3$, the qualitative behavior of the $N_{n} C_{n}(t)$ functions is not significantly affected by this spurious effect [10].

The $N_{n} C_{n}(t)$ functions for the systems studied in this work are shown in Figs. 1 and 2. According to earlier findings, the most characteristic features of these functions are as follows [10]. $N_{1} C_{1}(t)$ shows a fast initial increase that reflects the transfer of the initial momentum of the central particle to its immediate shell of neighbors. The later decay of $N_{1} C_{1}(t)$ should be associated with the spread of the momentum of the central particle to the outer shells. It may be observed in Figs. 1 and 2 (see the top plots) that the minima of the oscillatory $C(t)$ functions are located at the same position as the maxima of the corresponding $N_{1} C_{1}(t)$ functions 

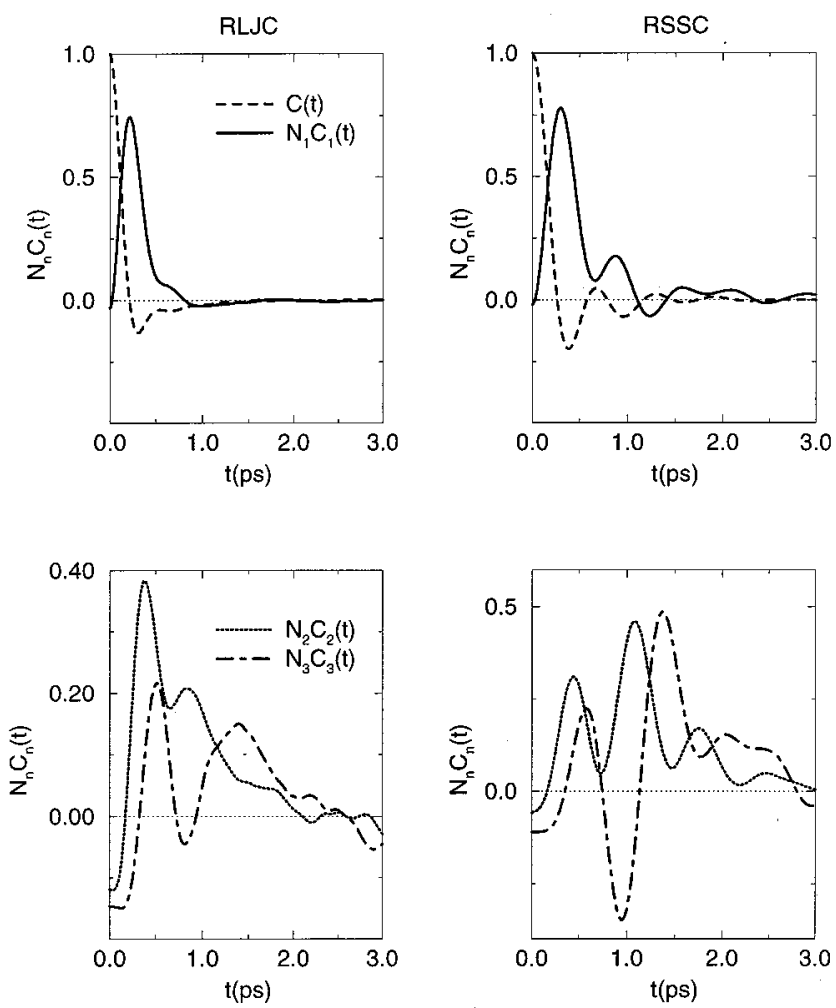

FIG. 2. Velocity cross-correlation functions in the systems at the state $C$ (high atomic density) for atoms within different coordination shells. The velocity autocorrelation functions are also shown in the upper part of the figure.

and vice versa. This indicates that in these systems part of the initial momentum transferred by the central particle to its neighbors is given back to this particle, bouncing back and forth for some time [1]. It should be noted that the initial rise of $N_{2} C_{2}(t)$ is shifted in relation to that of $N_{1} C_{1}(t)$ while the initial rise of $N_{3} C_{3}(t)$ is shifted in relation to that of $N_{2} C_{2}(t)$. These initial delays and the consequent shifts of the $N_{n} C_{n}(t)$ maxima should be attributed to the finite velocity of the propagation of momentum through the liquid. It may be noticed that both $N_{2} C_{2}(t)$ and $N_{3} C_{3}(t)$ start to rise before $N_{1} C_{1}(t)$ reaches its first maximum, which indicates that $N_{n} C_{n}(t)$ is the result of a balance between the momentum received from and/or transferred by particles in the $n$ shell. So, the process of transferring the initial momentum of the central particle to the different shells cannot simply be analyzed as the propagation of a wave through the liquid. As the density of the liquid increases the atoms are more closely packed and the velocities of distinct atoms more strongly correlated. Accordingly, the $N_{n} C_{n}(t)$ functions for RLJC and RSSC show larger values and more pronounced oscillations than those for RLJA and RSSA, respectively.

The comparison between the results for RLJ and RSS (Figs. 1 and 2) shows that the $N_{n} C_{n}(t)$ functions are markedly dependent on the softness of the potential core. Unlike for RLJ, all the $N_{n} C_{n}(t)$ functions for the RSS potential are oscillatory. Moreover, a tendency of the amplitude of the second oscillation to increase with $n$ may be observed. The pronounced oscillations of the $C(t)$ functions for potentials with soft cores are also clearly reflected in the corresponding $N_{1} C_{1}(t)$ functions. The existence of several maxima and minima in $N_{1} C_{1}(t)$ for both RSSA and RSSC indicates that there is a persistent interchange of momentum between the central particle and particles in the first coordination shell. This process requires a certain degree of coherence between the motion of atoms in the first coordination shell that is also revealed by the marked oscillations of the $N_{n} C_{n}(t)$ functions for $n>1$. It should be emphasized that even for a rather dilute liquid such as RSSA the $N_{n} C_{n}(t)$ functions show large oscillations. These findings suggest that collective motions are favored by potentials with soft cores, which is consistent with earlier results that indicate that longitudinal modes associated with density fluctuations propagate up to higher wave numbers in liquids with softer potential cores $[12,13]$. The results for the RLJ potential are very different. It is interesting to point out the presence of a shoulder after the first maximum in the $N_{1} C_{1}(t)$ function for RLJC while in the case of $N_{2} C_{2}(t)$ and $N_{3} C_{3}(t)$ there is a second maximum that becomes higher as $n$ increases. At low density (RLJA liquid), the shoulder is not visible but a second maximum may be noticed in both $N_{2} C_{2}(t)$ and $N_{3} C_{3}(t)$. The origin of a second maximum in $N_{n} C_{n}(t)$ will be discussed in Sec. II.

Both $C(t)$ and $N_{n} C_{n}(t)$ may be theoretically calculated according to the Gaskell and Miller velocity field approach [15], which is one of the simplest applications of the mode coupling concepts to the analysis of the dynamic properties in dense liquids. This approximation, which is a generalization of the hydrodynamic description of a fluid to microscopic scales, was successfully applied to LJ liquids, liquid alkali metals $[15,16]$, and, more recently, to the molecular center of mass of liquid water [17]. The theory is based on the introduction of a microscopic velocity field,

$$
\vec{\nu}(\vec{r}, t)=\sum_{j} f\left(\left|\vec{r}-\vec{r}_{j}(t)\right|\right) \vec{\nu}_{j}(t),
$$

where $f(r)$ is a form factor that may be determined by taking into account that the velocity field around the position of an atom should be very close to the actual velocity of this atom. Gaskell and Miller obtained an expression that allows us to calculate the velocity correlation functions from the selfintermediate scattering function $F_{S}(k, t)$ and the longitudinal $\left[C_{L}(k, t)\right]$ and transversal $\left[C_{T}(k, t)\right]$ current correlation functions $[1,15]$

$$
\begin{aligned}
C(t)= & \left(6 k_{B} \mathrm{Tm}\right)^{-1} \int_{0}^{\infty} f(k)\left[C_{L}(k, t)+2 C_{T}(k, t)\right] \\
& \times F_{S}(k, t) k^{2} d k,
\end{aligned}
$$

where $f(k)$ is the Fourier transform of $f(r)$. We assumed a form factor $f(r)=\exp \left[-(r / a)^{12}\right]$, where $a$ is an effective radius determined from the number density $\left(n_{\rho}\right)$ of the liquid according to the relation $4 \pi n_{\rho} a^{3} / 3=1$ [15]. The theory can also be applied to the calculation of the time correlation of the velocity of an atom $i$ with the sum of the velocities of the atoms within a sphere of center at $i$ and radius $R$ [18]:

$$
\phi_{R}(t)=\int_{0}^{R} F_{r}(t) d r,
$$



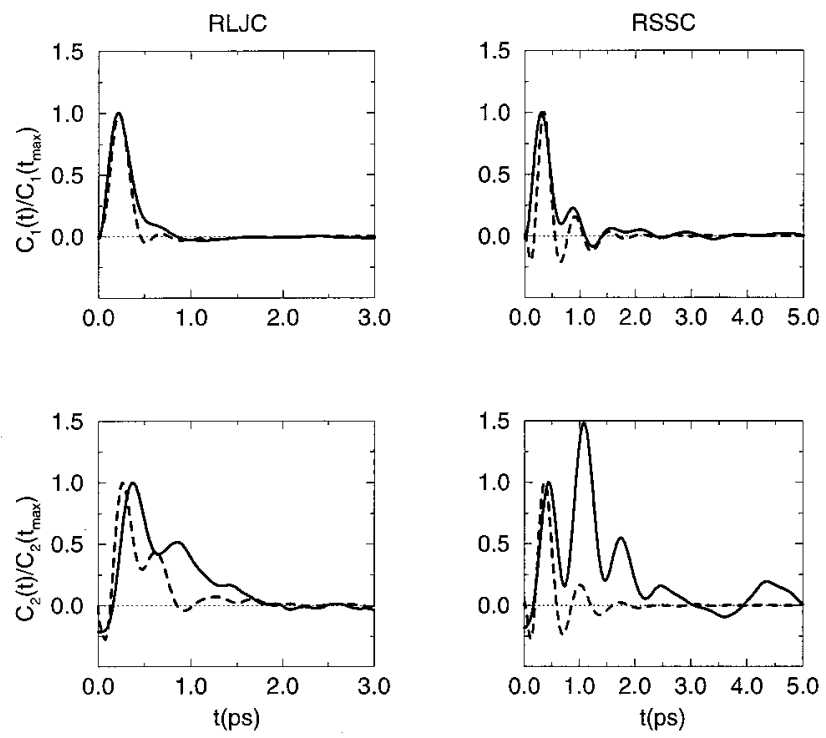

FIG. 3. Normalized velocity cross-correlation functions obtained from MD simulation (_ $)$ and calculated according to the velocity field model of Gaskell and Miller [15] (_-_-_).

where

$$
\begin{aligned}
F_{r}(t)= & 8 n_{\rho}\left(3 m k_{B} \mathrm{Tm}\right)^{-1} \int_{0}^{\infty} \sin (k r) f(k) \\
& \times\left[C_{L}(k, t)+2 C_{T}(k, t)\right] F_{S}(k, t) r k^{3} d k .
\end{aligned}
$$

The $N_{n} C_{n}(t)$ functions studied in this work may be calculated as the difference between two $\phi_{R}(t)$ functions with suitable radii.

We have calculated both the $C(t)$ and the $N_{n} C_{n}(t)$ functions for the RLJC and RSSC systems according to the velocity field model (this model is not valid for dilute fluids such as those at the state $A[13])$. The calculations in Eqs. (5) and (7) require one to integrate over $k$ a quantity that includes different dynamic collective properties $\left[F_{S}(k, t)\right.$, $C_{L}(k, t)$, and $\left.C_{T}(k, t)\right]$. We performed complementary MD simulations to determine these collective properties for a set of 20 wave numbers between $k=0.27$ and $k=4.4 \AA^{-1}$ [13]. For the sake of the $f(k)$ characteristics, the contributions to $C(t)$ corresponding to wave numbers beyond this interval are not significant. However, the finite number of $k$ 's used in the numerical integration did not allow us to accurately determine $C(t)$ and $F_{r}(t)$ through Eqs. (5) and (7). These inaccuracies may produce significant errors in the calculation of $N_{n} C_{n}(t)$ since these functions are obtained as the difference between two $\phi_{R}(t)$ functions for different radii.

The $C(t)$ functions obtained through Eq. (5) are in quite good agreement with the MD findings [13]. The agreement is better for RLJC than for RSSC since the theoretical $C(t)$ function for the second system shows an initial decrease slightly faster and more damped oscillations than those from MD. The $N_{n} C_{n}(t)$ functions calculated according to the velocity field theory and those directly obtained by MD show significant quantitative discrepancies that should be mainly attributed to numerical inaccuracies. Thus we have compared in Fig. 3 the $C_{n}(t)$ functions for $n=1$ and $n=2$ normalized to their first maximum $\left[C_{n}(t) / C_{n}\left(t_{\max }\right)\right]$. The disagreements
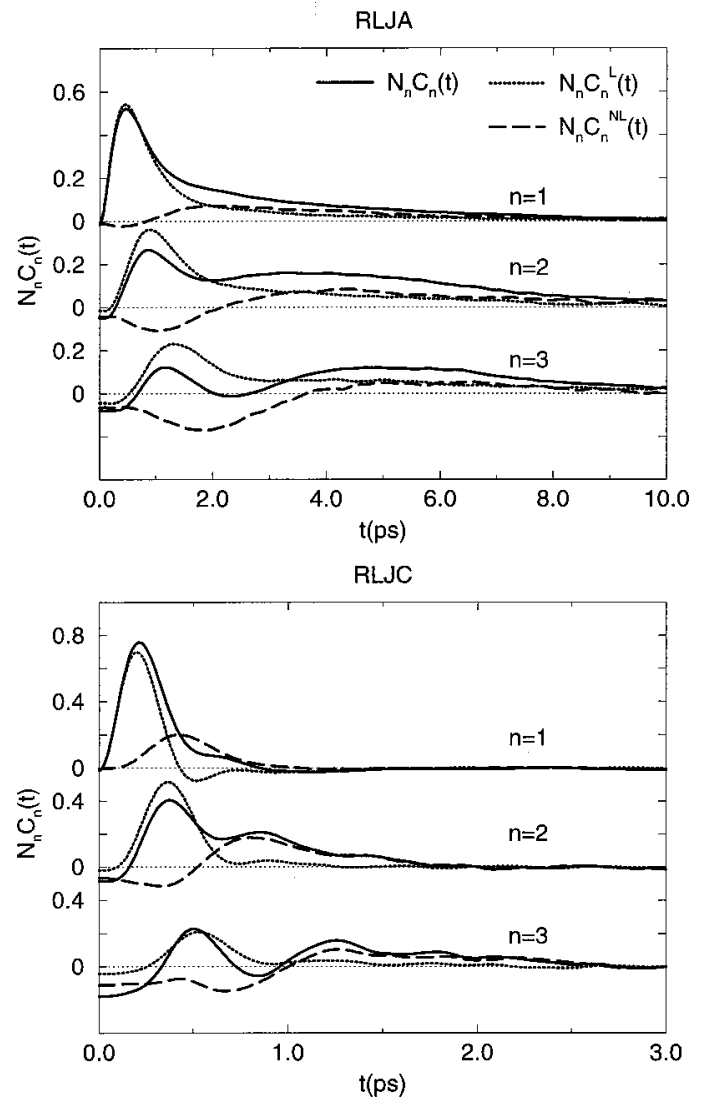

FIG. 4. Decomposition of the velocity cross-correlations corresponding to the "hard"' RLJ potential into longitudinal and nonlongitudinal contributions.

between theoretical and MD simulation results become larger as $n$ increases. The normalized $C_{1}(t) / C_{1}\left(t_{\max }\right)$ functions obtained through Eqs. (6) and (7) are quite similar to those from direct MD calculations whereas the discrepancies between the $C_{2}(t) / C_{2}\left(t_{\max }\right)$ functions by the two procedures are larger. The frequencies of the oscillations for RSSC, though not their relative amplitude, are well reproduced by theory. Dramatic differences between theoretical and MD results were found for $n=3$. A reliable quantitative calculation of the $C_{n}(t)$ functions for distant shells requires us to determine the collective dynamic properties in Eq. (7) for a very large set of $k$-wave numbers. This would require costly computer simulations beyond the objectives of this work [the direct calculation of $C_{n}(t)$ is much less expensive]. However, findings in this work show that the main qualitative features of the $C_{n}(t)$ functions in dense liquids are reproduced by the velocity field theory.

\section{LONGITUDINAL VELOCITY CROSS-CORRELATIONS}

In order to analyze with more detail the characteristics of the velocity cross-correlations, we have separately calculated the longitudinal $\left[C_{n}{ }^{L}(t)\right]$ and nonlongitudinal $\left[C_{n}{ }^{\mathrm{NL}}(t)\right]$ parts of the $C_{n}(t)$ functions:

$$
C_{n}(t)=C_{n}{ }^{L}(t)+C_{n}{ }^{\mathrm{NL}}(t) .
$$

$C_{n}{ }^{L}(t)$ is defined as the velocity cross-correlation along the direction defined by the centers of the particles $i$ and $j$ at $t$ $=0$. Thus $C_{n}{ }^{L}(t)$ was calculated according to [3] 

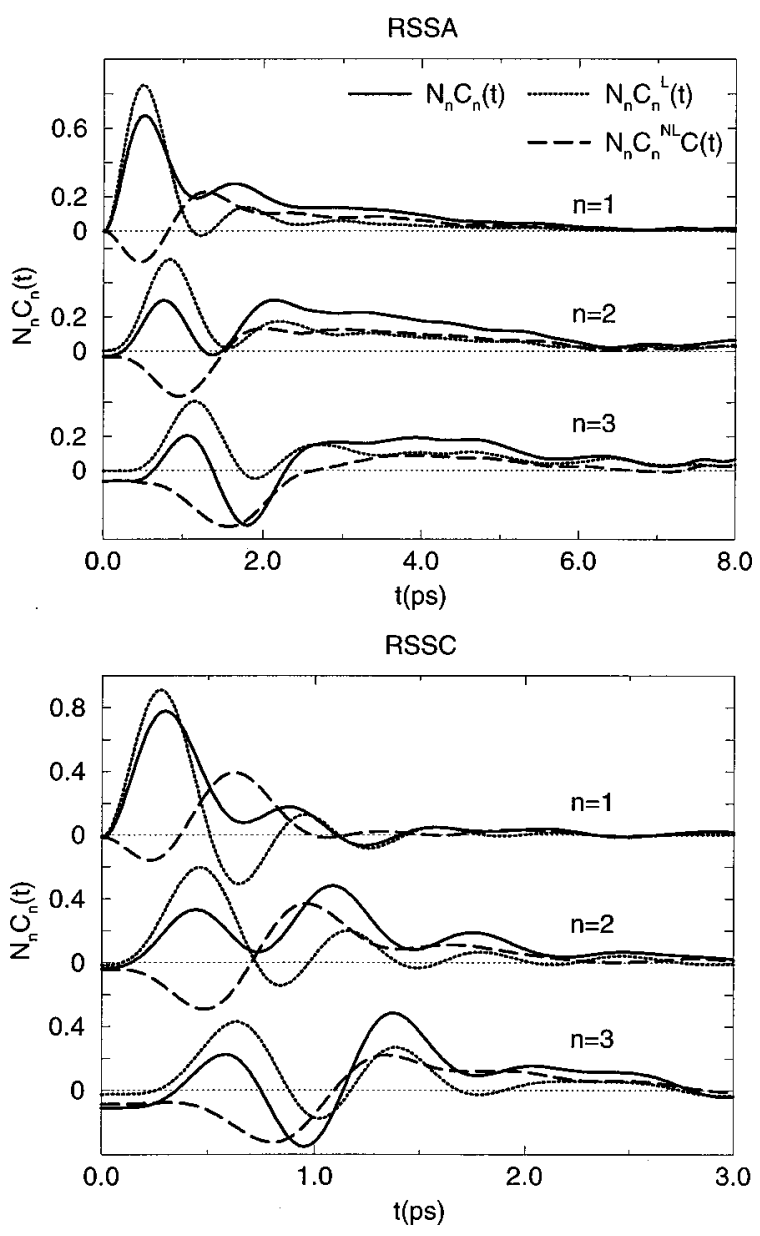

FIG. 5. Decomposition of the velocity cross-correlations corresponding to the "soft"' RSS potential into longitudinal and nonlongitudinal contributions.

$$
C_{n}{ }^{L}(t)=\left\langle\nu_{i}^{L}(0) \nu_{j}^{L}(t)\right\rangle_{R}\left\langle\nu_{i}^{2}\right\rangle^{-1}
$$

where $\nu_{i}{ }^{L}(0)=\vec{\nu}_{i}(0) \cdot \vec{r}_{i j}(0)$ and $\nu_{j}{ }^{L}(t)=\vec{\nu}_{j}(t) \cdot \vec{r}_{i j}(0)$ are the projections of the velocities of the particles $i$ and $j$ over the initial direction $i j . \vec{r}_{i j}(0)$ is a unitary vector along this direction. $C_{n}{ }^{\mathrm{NL}}(t)$ was determined from $C_{n}(t)$ and $C_{n}{ }^{L}(t)$ according to Eq. (8).

As could be expected, the short time velocity crosscorrelations between neighboring particles should be predominantly longitudinal. This is corroborated by the results in Figs. 4 and 5, which show that the initial part of $N_{1} C_{1}{ }^{L}(t)$ is very close to that of $N_{1} C_{1}(t)$. However, the $N_{n} C_{n}{ }^{\mathrm{NL}}(t)$ contribution to $N_{n} C_{n}(t)$ becomes significant at later times and for more distant shells. The shoulder of $N_{1} C_{1}(t)$ for RLJC as well as the second maxima in $N_{2} C_{2}(t)$ and $N_{3} C_{3}(t)$ for both RLJA and RLJC are absent in the corresponding $N_{n} C_{n}{ }^{L}(t)$ functions. These features of $N_{n} C_{n}(t)$ should then be attributed to correlations induced through the interactions with intermediate particles. As with $N_{1} C_{1}(t)$, the shoulder of the $C(t)$ function for RLJC should be associated with nonlongitudinal effects.

It may be thought that both $C_{1}(t)$ and $C_{1}{ }^{L}(t)$ are due to the direct collisions of the central particle with particles in front of it. However, the results of a previous study on the microscopic motions in simple liquids [6] suggested that cor-
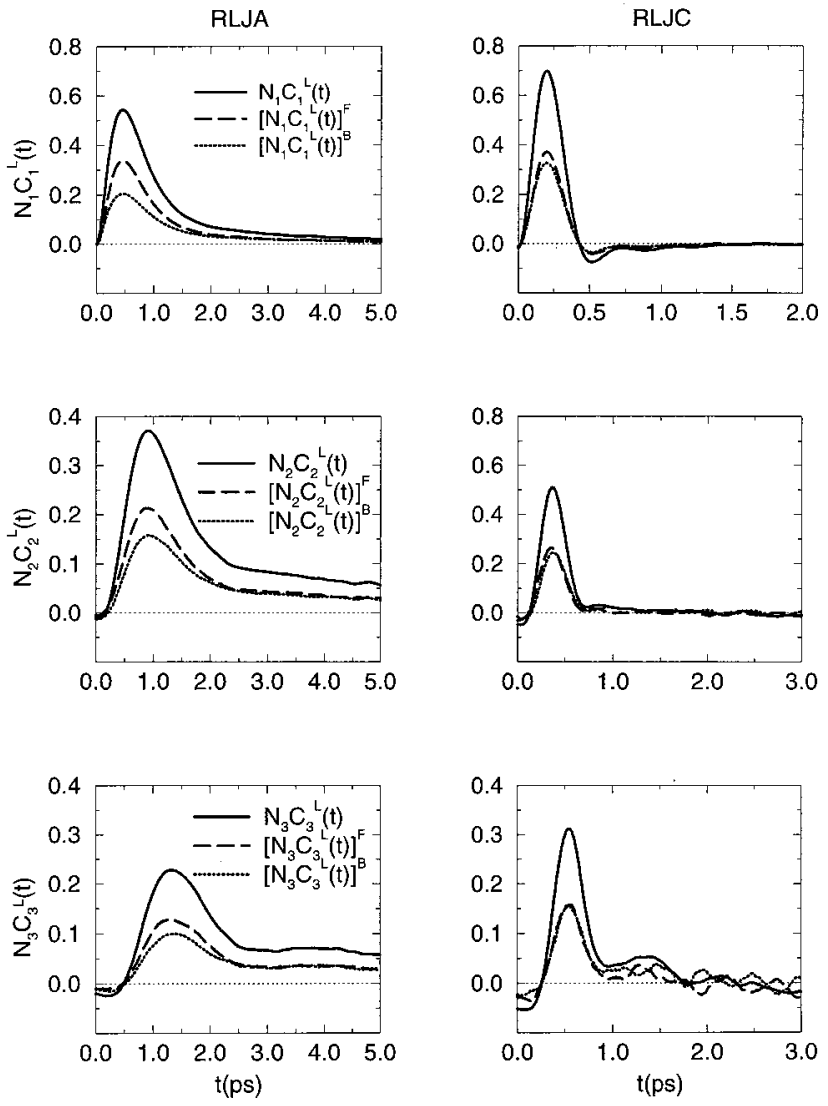

FIG. 6. Decomposition of the longitudinal velocity crosscorrelations into the forward and backward contributions (see the definitions in the text).

relations with the velocities of particles behind the tagged particle could also be important. In order to analyze this point we have divided the longitudinal velocity crosscorrelations into two contributions according to the sign of the initial velocity of particle $j$ with regard to the central particle $i$ :

$$
C_{n}{ }^{L}(t)=\left[C_{n}{ }^{L}(t)\right]^{F}+\left[C_{n}{ }^{L}(t)\right]^{B}
$$

The former term $\left[C_{n}{ }^{L}(t)\right]^{F}$ corresponds to the particles that at $t=0$ are approaching the central one, whereas the latter term $\left[C_{n}{ }^{L}(t)\right]^{B}$ corresponds to the particles that are initially moving away from the central particle. The results in Fig. 6 show that $\left[C_{n}{ }^{L}(t)\right]^{F}$ is almost identical to $\left[C_{n}{ }^{L}(t)\right]^{B}$ for RLJC and $\left[C_{n}{ }^{L}(t)\right]^{F}$ is somewhat larger than but of similar magnitude to $\left[C_{n}{ }^{L}(t)\right]^{B}$ for RLJA. These findings, which are consistent with those in [6], show that $C_{1}(t)$ and $C_{1}{ }^{L}(t)$ cannot only be attributed to the direct collisions of the central particle with particles found in its trajectory, and other mechanisms should contribute to the velocity crosscorrelation functions.

$\left[C_{1}{ }^{L}(t)\right]^{F}$ is associated with the momentum transfer due to the direct collisions of the central particle with a particle in its first shell of neighbors while $\left[C_{2}{ }^{L}(t)\right]^{F}$ and $\left[C_{3}{ }^{L}(t)\right]^{F}$ correspond to the momentum transferred through successive collisions with intermediate particles. However, the interpretation of $\left[C_{n}{ }^{L}(t)\right]^{B}$ is not so simple. It cannot be attributed to attractive forces between particles since the potentials in this 
study are purely repulsive. $\left[C_{1}{ }^{L}(t)\right]^{B}$ may be related to a tendency of particles behind the central particle to move in the same direction in order to fill the free space left by the migration of this particle. Then the local density is restored. These induced motions also propagate to more distant shells as reflected in the $\left[C_{n}{ }^{L}(t)\right]^{B}$ functions for $n=2$ and $n=3$. According to our interpretation, the importance of the $\left[C_{n}{ }^{L}(t)\right]^{B}$ terms should increase with density, which is corroborated by the smaller differences between $\left[C_{n}{ }^{L}(t)\right]^{F}$ and $\left[C_{n}{ }^{L}(t)\right]^{B}$ for RLJC in relation to those for RLJA.

The sign of the initial part of $N_{1} C_{1}{ }^{\mathrm{NL}}(t)$ is opposite to that of $N_{1} C_{1}{ }^{L}(t)$, being the location of the first minima in the former close to the first maxima in the latter. This is more clearly displayed in the case of the softer RSS potential. It should be noted that the first maximum of $N_{1} C_{1}{ }^{L}(t)$ for RSSA and RSSC is markedly higher than that of $N_{1} C_{1}(t)$ for the same system. However, it is interesting to point out that the two contributions tend to be on phase as the time increases and this causes the large second maxima in $N_{3} C_{3}(t)$. The initial negative values of the $C_{n}{ }^{\mathrm{NL}}(t)$ functions may be attributed to a mechanism similar to that suggested to explain the $\left[C_{n}{ }^{L}(t)\right]^{B}$ functions. When the central particle $i$ migrates with a velocity perpendicular to the $i j$ direction, the neighboring particles should move in the opposite direction in order to restore the local density. Thus the initial part of $C_{n}{ }^{\mathrm{NL}}(t)$, which should be mainly associated with these induced motions, is negative. Moreover, such motions produce local oscillations that propagate through the liquid and give rise to the second maxima in the $N_{n} C_{n}(t)$ functions for $n$ $>1$.

We want to emphasize the clear dependence of the $N_{n} C_{n}{ }^{L}(t)$ functions on the softness of the potential core. The $N_{n} C_{n}{ }^{L}(t)$ functions for both RSSA and RSSC show marked oscillations with very well defined maxima and minima and the deviations of the corresponding $N_{n} C_{n}(t)$ functions from regular oscillations are due to the $N_{n} C_{n}{ }^{\mathrm{NL}}(t)$ contributions. The frequency of the oscillations of the $N_{n} C_{n}{ }^{L}(t)$ functions for a given system is very similar for the different coordination shells but it may be observed to have a slight tendency to be lower as $n$ increases. The results for the RLJ potential are very different from those for the RSS potential. The $N_{n} C_{n}{ }^{L}(t)$ functions for both the RLJA and RLJC systems do not show any noticeable oscillation and are practically reduced to a rather fast initial rise followed by a slower decay. The velocity cross-correlations only reach negative values and show an incipient oscillation in the case of RLJC with $n=1$. The noticeable oscillations of $N_{2} C_{2}(t)$ and $N_{3} C_{3}(t)$ for the RLJ systems should be related to the nonlongitudinal contributions.

\section{CONCLUDING REMARKS}

Findings in this paper show that velocity crosscorrelations and momentum transfers between distinct particles cannot only be associated with direct interactions such as two-body collisions, but should also be related to the velocities induced by the motions of a tagged particle on its neighbors to restore the local density. This reflects a close relationship between velocity cross-correlations and density fluctuations. The longitudinal velocity cross-correlations (as defined in this paper) are markedly oscillatory for potentials with soft cores. These oscillations are related to collective motions of close particles that propagate through the liquid. It should be noted that the propagation of density fluctuations (longitudinal modes) in liquids is enhanced by potentials with soft cores, as proved by the persistence of up to rather high wave numbers of the Brillouin peak in the dynamic structure factor of liquid metals $[12,14]$. According to the suggestion of Lewis and Lovesey [19], our results indicate that the large-angle scatterings produced by hard core interactions are particularly effective in destroying the coherence necessary for collective motions of atoms. Finally, we want to emphasize the noticeable influence of the nonlongitudinal terms on the qualitative features of both $C(t)$ and $N_{n} C_{n}(t)$ functions at intermediate times.

\section{ACKNOWLEDGMENTS}

Financial support by the DGICYT (Grant No. PB960170-C03) and the CIRIT (Grant No. GRR 93-3019) is gratefully acknowledged.
[1] U. Balucani and M. Zoppi, Dynamics of the Liquid State (Clarendon, Oxford, 1994).

[2] U. Balucani, R. Vallauri, and C. S. Murthy, Phys. Lett. 84A, 133 (1981).

[3] U. Balucani, R. Vallauri, and C. S. Murthy, J. Chem. Phys. 77, 3233 (1982).

[4] U. Balucani, R. Vallauri, C. S. Murthy, T. S. Gaskell, and M. S. Woolfson, J. Phys. C 16, 5605 (1983).

[5] U. Balucani, R. Vallauri, T. Gaskell, and M. Gori, Phys. Lett. 102A, 109 (1984).

[6] Y. Endo and H. Endo, J. Chem. Phys. 80, 2087 (1984).

[7] D. M. Heyes and W. C. Sandberg, Phys. Chem. Liq. 22, 31 (1990).

[8] P. Flener and F. J. Veseley, Mol. Phys. 77, 601 (1992).

[9] W. C. Sandberg and D. M. Heyes, Mol. Phys. 85, 635 (1995).
[10] A. Verdaguer, J. A. Padró, and J. Trullàs, J. Chem. Phys. 109, 228 (1998).

[11] A. Verdaguer, J. A. Padró, and J. Trullàs, J. Mol. Liq. (to be published).

[12] M. Canales and J. A. Padró, Phys. Rev. E 60, 551 (1999).

[13] N. Anento, J. A. Padró, and M. Canales, J. Chem. Phys. 111, 10210 (1999).

[14] J. P. Hansen and I. R. McDonald, Theory of Simple Liquids, 2nd ed. (Academic, London, 1986).

[15] T. Gaskell and S. Miller, J. Phys. C 11, 3749 (1978).

[16] T. Gaskell and S. Miller, J. Phys. C 11, 4839 (1978).

[17] U. Balucani, J. P. Brodholt, and R. Vallauri, J. Phys.: Condens. Matter 8, 6139 (1996).

[18] T. Gaskell and P. E. Mason, J. Phys. C 14, 561 (1981).

[19] J. W. E. Lewis and S. W. Lovesey, J. Phys. C 10, 3221 (1977). 